PSICOLOGÍA

IBEROAMERICANA
Psicología Iberoamericana ISSN: 1405-0943

revista.psicologia@ibero.mx

Universidad Iberoamericana, Ciudad de México México

\title{
Impacto psicosocial e intervenciones de apoyo para cuidadores informales de personas dependientes en méxico
}

Hurtado-Vega, Juan Carlos

Impacto psicosocial e intervenciones de apoyo para cuidadores informales de personas dependientes en méxico

Psicología Iberoamericana, vol. 29, núm. 1, 2021

Universidad Iberoamericana, Ciudad de México, México

Disponible en: https://www.redalyc.org/articulo.oa?id=133967526007

DOI: https://doi.org/10.48102/pi.v29i1.339

(c) (1)

Esta obra está bajo una Licencia Creative Commons Atribución 4.0 Internacional. 
Artículos

\section{Impacto psicosocial e intervenciones de apoyo para cuidadores informales de personas dependientes en méxico}

Psychosocial impact and support interventions for informal caregivers of dependent people in Mexico

Juan Carlos Hurtado-Vega juan.hurtado@ibero.mx Universidad Iberoamericana, Ciudad de México, México

https://orcid.org/0000-0003-4984-3861

Psicología Iberoamericana, vol. 29, núm. 1,2021

Universidad Iberoamericana, Ciudad de México, México

Recepción: 05 Febrero 2021 Aprobación: 26 Mayo 2021

DOI: https://doi.org/10.48102/ pi.v29i1.339

Redalyc: https://www.redalyc.org/ articulo.oa?id=133967526007
Resumen: El 41\% de los mexicanos mayores de 60 años con alguna dependencia están siendo atendidos durante la pandemia de COVID-19 por un cuidador informal. El perfil más representativo de cuidador informal mexicano corresponde a una persona de sexo femenino, mayor de cincuenta años, hija o cónyuge del dependiente. Los cuidadores informales dedican catorce horas diarias a cuidar, presentan más ansiedad y depresión, y peor salud física que los no-cuidadores. El presente ensayo advierte sobre la pertinencia de revisar el impacto psicosocial y diversas propuestas de intervención para apoyar necesidades específicas de los cuidadores informales. Se concluye que las mejores propuestas deben ser contextualizadas, breves, sostenidas en técnicas eficaces (tales como: intervenciones grupales, basadas en premisas cognitivo-conductuales que fomenten la participación del cuidador), metodológicamente explícitas, y accesibles en términos económicos. $\mathrm{Al}$ conocer sus circunstancias, los no-cuidadores identificarán diferentes tipos de impactos psicosociales en los cuidadores, y las mejores formas de ayudarles.

Palabras clave: cuidadores informales, personas dependientes, impacto psicosocial, estrategias de intervención.

Abstract: Forty-one percent of Mexicans over 60 years of age with a dependency condition are being cared by an informal caregiver during the Covid-19 pandemic. The most representative profile of a Mexican informal caregiver is a woman who is more than 50 years old and is the daughter or spouse of the dependent person. Informal caregivers spend 14 hours a day providing informal care, and experience more symptoms of anxiety and depression, and worse physical health than non-caregivers. This critical essay calls attention to the psychosocial impact of informal and unpaid care work and the various possible interventions for informal caregivers. The best solutions must be contextualized, concise, based on effective techniques (such as, group proceedings, based on cognitive-behavioural premises that promote the participation of the caregiver), methodologically explicit, and financially accessible. This essay allows readers to see the psychosocial impacts informal unpaid care work has on these caregivers and the best ways to help them.

Keywords: informal caregivers, dependent people, psychosocial impact, intervention proposals.

\section{Introducción}

Los habitantes de México están acelerando sus índices de envejecimiento. Esta afirmación se sostiene en conformidad con los datos obtenidos en el último censo oficial de población, en donde se señala que los mayores 
de 60 años representan al 12\% de los habitantes del país (INEGI, 2020). Es importante señalar que la expectativa de vida de los mexicanos ha aumentado con respecto a 15 años atrás: en el presente, en promedio es de 77.4 años para las mujeres y de 71.7 años para los hombres (ValdésCorchado et al., 2020).

Se espera que para el año 2030 el porcentaje de adultos mayores se incremente hasta representar al $14.8 \%$ de la población nacional: así fue proyectado en una investigación llevada a cabo en la Ciudad de México, cuyo tema fue la asociación de la fragilidad fenotípica (sarcopenia, dinapenia) con la presencia de abuso físico, psicológico y/o sexual en los adultos mayores (Torres - Castro et al., 2018).

Ante esta configuración etaria del país, cabe preguntarse: ¿Quiénes son las personas que se hacen cargo de una población envejecida que va en aumento, con arduas necesidades de cuidado, y bajo una difícil situación de dependencia?

\section{Los cuidadores informales de personas dependientes}

Según datos proporcionados por el Banco Interamericano de Desarrollo, el $25 \%$ de las personas adultas mayores en México necesitan cuidados informales en el hogar (López-Ortega \& Aranco, 2019). Las razones para necesitar estos cuidados obedecen a circunstancias que se desarrollan a largo plazo, tales como la presencia de enfermedades crónicas o situaciones de discapacidad funcional (López-Ortega \& Aranco, 2019).

El Instituto Nacional de Geriatría de México reportó la existencia de $1,200,794$ personas mayores dependientes, es decir, con algún tipo de limitación para ejecutar actividades básicas de la vida diaria, que contaron con la asistencia de por lo menos un cuidador durante el año 2019 (INGER, 2019).

En México la obligación del cuidado formal e informal de los enfermos ha recaído principalmente en la población femenina. Así lo muestra la Encuesta Nacional sobre Uso del Tiempo realizada en el año 2009, en la que se encontró que 1,239,530 mujeres y 861,832 hombres refirieron brindar apoyo y cuidado a personas dependientes (INEGI, 2009).

El que sean mayoritariamente mujeres las personas que se dedican al cuidado, corresponde a un hecho que se asume tanto por una realidad sociodemográfica propia del país, como por antiguas y muy extendidas tradiciones culturales y corrientes ideológicas (Rangel et al., 2017).

En nuestro canon occidental, y debido al influjo de diversas fuerzas sociales, han sido las integrantes del sexo femenino quienes históricamente asumieron el rol de cuidadoras y poseedoras de los conocimientos sobre la atención a la salud. Para ilustrar este hecho se menciona que, en nuestro país, las personas dedicadas a la actividad de la enfermería, ya sea informal o profesionalmente, son en su mayor parte del sexo femenino (Tello de la Torre \& Vargas Villamizar, 2020).

Además, las mujeres suelen hacerse cargo del acompañamiento de los enfermos en cualquier sistema de salud al que éstos deban, o puedan, acudir (Barragán Solis, 2007). 
Es interesante consignar que, de acuerdo con una investigación mexicana sobre autopercepción de eficacia para llevar a cabo cuidados, resulta que son mujeres las que más auto-eficaces se perciben para cuidar a una persona dependiente, en contraste con la menor percepción de autoeficacia para cuidar que reportaron los varones (Muñoz, 2019).

Con todo esto es posible definir el perfil más representativo de cuidador informal mexicano: corresponde con el de una mujer, con una edad promedio superior a 50 años, casada, que suele ser la hija o la cónyuge de la persona que precisa de apoyo (INMUJERES, 2008).

En promedio, la cuidadora informal mexicana suele dedicar 14 horas diarias a la atención de un dependiente $(\mathrm{DE}=7.57)$, tal y como fue registrado en una investigación que asoció la sobrecarga de los cuidadores y su calidad de vida, con la funcionalidad familiar (Salazar-Barajas et al., 2019).

Las cuidadoras informales sólo tienen otro trabajo -además de cuidaren el $29 \%$ de los casos, y se calcula que $74 \%$ de ellas se encuentran sin apoyo de otras personas o profesionales médicos (Muñoz, 2019).

Las y los cuidadores informales no suelen tener una formación en temas de atención a la salud, y usualmente no reciben una remuneración económica por sus cuidados. Quienes adquieren -ya sea por voluntad propia o a pesar suyo- la función de cuidar, cumplen con este papel sin someterse a horarios ni a capacitaciones formales. Pasan gran parte del día con la persona que cuidan y en muchas ocasiones conviven con ella. La ayuda que suministran es constante e intensa, y algunas veces dicha atención sobrepasa su capacidad física y mental. Todas las condiciones arriba señaladas, conforman un nuevo evento estresante crónico, además del producido por atestiguar el progresivo deterioro funcional de un ser querido (Trujillo Ornelas \& Martínez, 2019).

En no pocos casos la ansiedad y el estrés del cuidador desembocan en maltrato, voluntario o involuntario, a la persona dependiente: durante el 2016 se reportó que la prevalencia de maltrato a personas mayores fue de $10.3 \%$, y afectó a un $32 \%$ de los adultos mayores con alguna discapacidad. Los anteriores fueron datos clasificados como "hechos destacados acerca del envejecimiento y la vejez en México", en un reporte del Instituto Mexicano de Geriatría sobre la situación de los adultos mayores mexicanos (Robledo et al., 2016, p. 44).

Por todo lo anterior, el presente ensayo advierte sobre la necesidad de revisar las dimensiones y los alcances del impacto psicosocial producido en el cuidador informal, como resultado del ejercicio de sus actividades. Del mismo modo, en este escrito se revisará la eficiencia de algunas propuestas de intervención con cuidadores, que se han diseñado para tratar de apoyar y atender su amplia gama de necesidades. También se pretende contribuir a la divulgación (dirigida tanto a no-cuidadores, como a los distintos tipos de cuidadores) del vasto panorama de temas de prevención, psicoeducación, asistencia emocional, y capacitación técnica de las personas que se dedican a cuidar.

En consecuencia, y tal y como se desprende de las conclusiones emitidas por un artículo español de revisión sistemática que analizó temas de ayuda 
brindada a cuidadores en la década de los noventa, se ha encontrado que los factores que tienen un papel fundamental en las necesidades de auxilio a los cuidadores informales son: 1) La ubicación, accesibilidad económica, capacidad y profesionalismo de las instituciones privadas y/o públicas de cuidados a personas dependientes; 2) El tipo y grado de pérdida de autonomía de la persona que recibe los cuidados; 3) El estado de salud, la fuerza emocional y el bienestar físico del cuidador; 4) El nivel económico, el aislamiento geográfico, el aislamiento social, y la participación o no en el mundo laboral por parte de la o el cuidador informal; 5) La ausencia o presencia de otros cuidadores; 6) El compartir o no la misma residencia con la persona cuidada; 7) En algunos casos, el sexo del cuidador aparejado al sexo de la persona dependiente (Escuredo, 2007).

En lo que ha sido posible hallar sólido consenso, es en la afirmación de que la mayoría de las y los cuidadores informales necesitan mucho más de lo que reciben, no importando si las ayudas provienen de instituciones públicas, privadas, o de individuos más o menos familiarizados con los cuidados. De manera abrumadoramente mayoritaria, las necesidades a las que los cuidadores suelen hacer referencia son: el descanso, la ayuda económica, el apoyo psicológico y formativo, así como las facilidades para compatibilizar el cuidado y el trabajo remunerado, de acuerdo con lo reportado en la investigación ya referida en el párrafo anterior, en donde se revisó de manera sistemática la evolución de las políticas públicas de protección social a personas dependientes (Escuredo, 2007).

Se ha observado que, tanto a las personas dependientes, a sus familias, así como a las instituciones públicas y privadas de atención a la salud, les interesa mantener la actividad de cuidar en el entorno comunitario, y aún hogareño. Sin embargo, la escasez o insuficiencia de los recursos de apoyo para las y los cuidadores informales convierten al escenario de brindar cuidados en un desafío muy demandante. Así, y como un derivado de estas dos condiciones, las personas que cuidan acaban desarrollando niveles de malestar psicológico y físico que les obstaculizan o impiden ejercer sus actividades de cuidado de una forma adecuada y adaptativa tanto para ellas, como para las personas dependientes (Pabón Poches et al., 2014).

Hasta ahora, los cuidadores han sido considerados como un recurso para subsanar problemáticas públicas, sociales y/o familiares, pero apenas se piensa en ellas y ellos como beneficiarios directos de ayudas gubernamentales (Vázquez et al., 2010).

Desde un punto de vista teórico y metodológico, las personas que cuidan y sus circunstancias se han considerado como constructos definidos operativamente de manera incompleta, no actualizados, y que se caracterizan por la imprecisión conceptual en todas sus facetas (Saavedra et al., 2020).

Debido a lo cual, se puede adelantar ya una propuesta para robustecer el estudio de los cuidadores informales: investigaciones con más alta especificidad en sus objetivos metodológicos fomentarían una comprensión más cabal de las condiciones de existencia del cuidador. $\mathrm{Si}$ los estudios fueran metodológicamente más precisos, se podrían realizar comparaciones sistemáticas y establecer criterios de calidad en 
las intervenciones de ayuda. Con los resultados de estas investigaciones, que ahora podrían ser medibles y equiparables, sería factible contribuir a una mejor y más profunda comprensión de las experiencias cotidianas que viven las y los cuidadores, disminuyendo así el impacto personal que produce el gestionar cuidados a personas dependientes.

\section{Representaciones psicológicas acerca del cuidador informal}

En un estudio sobre representaciones psicológicas y asistencia a personas realizado en México por Díaz et al. (2019), se acredita la creencia generalizada de que los adultos mayores merecen más cuidados y atenciones. Sin embargo, las mismas personas que suscriben dicha creencia no le otorgan reconocimiento especial a las personas que brindan cuidados, en el sentido de que no valoran su actividad como una acción señaladamente representativa de amor y sacrificio. Es decir: según el criterio mayoritario recopilado en la investigación que se acaba de referir, toda la carga de actividades que llevan a cabo las y los cuidadores no representa otra cosa más que una respuesta natural, esperada y obligada, que se desprende de los compromisos familiares.

Más aún: las mismas personas que brindan cuidados tienden a no percibir las acciones que hacen como difíciles, laboriosas, especiales o pesadas (Gutiérrez Cuéllar, 2019).

Retomando la investigación sobre representaciones psicológicas citada más arriba: en ella se reportó, en una cohorte de cuidadoras informales, el registro de una percepción de no-carga relativa a sus actividades de cuidado. Sin embargo, también se encontró que esta misma percepción de no-carga estuvo asociada a la comisión de agresiones psicológicas dirigidas hacia las personas dependientes que eran asistidas. Es decir: en la cohorte de cuidadoras informales analizada, aunque las actividades de cuidado no fueron percibidas como conflictivas o difíciles, se tuvo la representación psicológica de que las personas dependientes a su cargo sí eran problemáticas y confrontativas (Díaz et al., 2019).

El anterior es un ejemplo de los múltiples intercambios emocionales que tienen lugar entre el cuidador informal y la persona asistida al estar en interacción. Desgraciadamente no todas las dinámicas de cuidado resultan siempre ser psicológicamente armónicas, y el intercambio mutuo de intensos afectos puede dejar como resultado todo tipo de impactos negativos en ambos integrantes.

\section{Impacto social y laboral en el cuidador informal}

Los cuidadores informales que no tienen un empleo público experimentan importantes afectaciones debido a los costos económicos ocasionados por el tiempo que se dedica al cuidado de una persona dependiente en casa, ya sea porque disminuyen los ingresos, o porque aumentan los gastos derivados de los cuidados administrados (Montes et al., 2016). 
Para analizar esta situación, es preciso tener en cuenta si acaso la relación entre el cuidado informal y la participación en el mercado de trabajo formal es endógena. Esto es: si los y las trabajadoras dejaron de laborar oficial y administrativamente fuera del hogar para convertirse en cuidadores informales, puesto que de esta forma la afectación económica resulta ser mayor (Moya-Martínez et al., 2012);

El tiempo invertido en cuidar a otros, de manera directa implica que las y los cuidadores dejan de disponer de espacios personales dedicados a actividades sociales, de ocio, o de autocuidado, en detrimento de su propia salud física y psicológica. A largo plazo, esta situación aumenta el gasto de dinero destinado a cuestiones médicas, el número de pacientes dentro de un mismo núcleo familiar, y la necesidad de más cuidadores informales y/o formales para un clan que no está preparado para producirlos ni contratarlos (Montes et al., 2016).

\section{Impacto psicológico en el cuidador informal}

Múltiples investigaciones indican que el cuidado informal supone una fuente de estrés crónico que puede tener graves consecuencias sobre la salud física y mental, como ha quedado asentado en la revisión sistemática sobre intervenciones de ayuda a cuidadores informales de mayores con demencia no-institucionalizados, realizada por Bustillo et al. (2018), en un periodo comprendido entre los años 2005 al 2016.

En la mayoría de los casos el parentesco de la o el cuidador con la persona dependiente hace más emocional, y como consecuencia más difícil, el brindar cuidados (Muñoz, 2019).

Los cuidadores son quienes reciben todas las emociones ambivalentes de otros familiares no-cuidadores de la persona dependiente, lo que significa que experimentan gratitud, pero también enojo y culpa por el sufrimiento del familiar a su cargo, o por el agravamiento de sus enfermedades. Las y los cuidadores se encuentran encarando los malestares físicos de las personas a las que cuidan, pero también todas sus expectativas y sus frustraciones. Asimismo, son el blanco de la crítica, justificada e injustificada, de parte de la familia extendida de la persona dependiente. Todas las circunstancias que se acaban de señalar, más los problemas físicos, psicológicos y sociales propios del cuidador, generan intensos cambios que se manifiestan en impactos y consecuencias emocionales difíciles de predecir, y que ponen a prueba la resiliencia del grupo familiar (Salazar-Barajas et al., 2019).

En la revisión sistemática sobre intervenciones en cuidadores de mayores con demencia no-institucionalizados realizada en España, y que fue mencionada más arriba, se encontró que las y los cuidadores informales, analizados en grupos que iban de los 32 a los 260 participantes, presentaron niveles significativamente mayores de ansiedad y depresión que los demás integrantes de la familia que eran no-cuidadores, así como un peor estado de salud física (Bustillo et al., 2018).

Dentro de las consecuencias psicológicas y psicosomáticas más comunes en las y los cuidadores informales se encuentran: 1 . Alteraciones 
del sueño. 2. Depresión. 3. Estrés. 4. Dolor articular. 5. Cefaleas. 6. Conductas e ideación suicida. 7. Agresividad. 8. Abuso de sustancias. 9. Inatención. 10. Baja autoestima. 11. Deseos de abandonar el trabajo (y el cuidado). 12. Negación de las emociones o desplazamiento de afectos. $\mathrm{Y}$, finalmente, 13. Una peor respuesta inmune a la influencia de vacunas, asunto de la mayor importancia en medio de una pandemia (Montes et al., 2016).

Además, son característicos por parte de las y los cuidadores informales los reportes de sentimientos de culpa, indefensión, frustración, negación de la necesidad de ayuda, e ideas de soledad (Montes et al., 2016).

Las consecuencias reseñadas en los dos párrafos anteriores fueron detalladas en una revisión sistemática de estudios descriptivos y correlacionales, en la que se mostró que existe una conexión entre la afectación de la salud física y psicológica de las y los cuidadores, con el hecho de brindar cuidados informales (Montes et al., 2016).

Por otro lado, se ha encontrado que los cuidadores que reciben ayudas externas de familiares o de trabajadores sociales presentan un nivel intermedio de ansiedad (Mercedes \& Rivera, 2018).

De manera complementaria, se ha registrado que los cuidadores que tienen menos ansiedad son los que tienen ayuda efectiva de profesionales médicos (Mercedes \& Rivera, 2018).

Los dos últimos datos consignados, relativos a la presencia de ansiedad en los cuidadores, fueron publicados en una investigación española que tuvo como objetivo explorar la relación que mantienen los cuidadores informales con su familiar dependiente, ya ingresado en alguna residencia o institución de ayuda (Mercedes \& Rivera, 2018).

A continuación, se presenta una serie de datos reportados en una investigación llevada a cabo en España que pretendió averiguar los niveles de ansiedad y autoeficacia en cuidadores de enfermos de Alzheimer (Muñoz, 2019): Las y los cuidadores que cuentan con bachillerato presentan mayor ansiedad que los de formación universitaria o formación básica. Los que llevan de 0 a 3 años como cuidadores son los que presentan mayor ansiedad, y se van reduciendo estos registros a lo largo de los años, llegando a puntuar con la menor tasa de ansiedad los que llevan más de diez años cuidando a la persona dependiente. Las y los cuidadores informales que dedican dos horas o menos a los cuidados son los que más ansiedad presentan, seguidos por los cuidadores que dedican entre 5 a 7 horas a sus actividades. Los que menos ansiedad presentan son los que dedican más de diez horas diarias a la labor de cuidador. Sin embargo, los cuidadores veteranos que ya casi no presentan ansiedad originada por la ejecución rutinaria de sus actividades poseen índices más altos de agotamiento físico y burn-out, que sus contrapartes menos experimentadas.

La conclusión del estudio al que se ha hecho referencia en el párrafo anterior indica que las variables de ansiedad y autoeficacia en los cuidadores informales están moduladas conforme al tiempo que éstos últimos han dedicado a los cuidados (Muñoz, 2019). 
Si esta variedad de impactos psicológicos no se atiende de manera adecuada, las condiciones y las circunstancias de las y los cuidadores informales los encaminarán a presentar síndrome de sobrecarga.

\section{Sobrecarga del cuidador informal}

En la revisión sistemática sobre intervenciones en cuidadores de mayores con demencia ya referida más arriba, se encontró que la notable -pero generalmente inadvertida para los no-cuidadores- inversión de tiempo, y la complejidad operativa que requieren las tareas del cuidado, son consideradas las causas principales de la sobrecarga del cuidador (Bustillo et al., 2018).

El cuidador informal va asumiendo poco a poco tantas responsabilidades como autonomía va perdiendo la persona dependiente, puesto que las actividades del cuidado gravitan en torno al avance progresivo e imparable de enfermedades crónico-degenerativas (Muñoz, 2019).

De acuerdo con un estudio mexicano de carácter descriptivo y correlacional con muestreo de tipo bola de nieve, llevado a cabo en centros comunitarios del país, la sobrecarga del cuidador es considerada multidimensional, con características objetivas y subjetivas: objetivas, por la demanda específica de energía que representan las actividades del cuidador, y subjetivas, por aquellas reacciones de tipo emocional provocadas por la realidad concreta del cuidado (Salazar-Barajas et al., 2019).

Como ejemplo de las características subjetivas de la sobrecarga, se consigna que las y los cuidadores no suelen reconocer el tremendo impacto de las actividades de asistencia sobre su propio estado de salud y su calidad de vida. La falta de auto-reconocimiento los lleva a empeorar su condición debido a que, para ellos, la máxima prioridad es el cuidado del otro, y no el propio. Las inquietudes de los cuidadores suelen disminuir, pero no terminan por erradicarse y aún son redirigidas a temas prácticos del cuidado, únicamente en el momento en que están atendiendo a la persona dependiente (Salazar-Barajas et al., 2019).

En el estudio descriptivo-correlacional arriba citado, también se encontró que las principales manifestaciones emocionales de la sobrecarga del cuidador están representadas por la continua presencia de estados depresivos y de ansiedad (Salazar-Barajas et al., 2019).

Los investigadores mexicanos que participaron en la investigación de marras señalan que los principales problemas físicos debidos a la sobrecarga del cuidador son: cefalea, astenia, fatiga crónica, y alteración del ciclo del sueño (Salazar-Barajas et al., 2019).

Para otorgar contexto al estudio al que se ha venido haciendo referencia, se comenta que tenía por objetivo asociar la sobrecarga de los cuidadores y su calidad de vida, con la funcionalidad familiar. Hay que señalar que los cuidadores que integraron la muestra de trabajo en dicha investigación tenían al menos 6 meses al cuidado de una persona 
dependiente y el 56\% de ellos presentaban sobrecarga (Salazar-Barajas et al., 2019).

En otro orden de ideas, es importante apuntar que los enormes retos que cotidianamente experimentan los y las cuidadoras se agravan en entornos rurales donde no hay servicios de asistencia accesibles, ni especializados en distintos tipos de enfermedades crónicas y/o dependencias (Agulló Tomás et al., 2019).

El principal impacto de todas estas condiciones consiste en que el síndrome de sobrecarga está asociado con el riesgo de maltrato a la persona dependiente (Montes et al., 2016).

Sin embargo, no todas las y los cuidadores informales presentan síndrome de sobrecarga, ni cometen maltratos a la persona que cuidan. Resulta pertinente entonces, señalar cuáles pueden ser los factores de riesgo que tienen el potencial de patologizar el vínculo físico y afectivo que se establece al brindar cuidados.

Así tenemos que, dentro de los factores de riesgo demográficos, las mujeres cuidadoras presentan mayores niveles de depresión, ansiedad, sintomatología psiquiátrica, y peor percepción de bienestar subjetivo que los cuidadores varones (Rangel et al., 2017).

Por otro lado, también son factores de riesgo para sobrecarga: 1.) Ser cuidador de mediana edad. 2.) El bajo nivel de escolaridad. 3.) Tener recursos económicos desfavorables. 4.) Permanecer soltero. 5.) La percepción negativa sobre el estado de salud de la persona cuidada. 6.) El grado de dependencia de la persona asistida 7.) La utilización inadecuada de los estilos y estrategias de afrontamiento del cuidador. 8.) La insatisfacción con el apoyo percibido de la red social. 9.) La existencia de un vínculo desfavorable con el familiar que es apoyado. 10.) La actitud negativa del enfermo hacia el tratamiento y el cuidado. Y, finalmente, 11.) Enfermedades en la persona dependiente que afecten la cognición y el comportamiento (Montes et al., 2016).

\section{Estrategias de intervención para el cuidador informal}

Las y los cuidadores que se perciben solos, sin ayuda, padeciendo dificultades económicas, sociales, y que no tienen capacitación formal en temas de cuidado de personas dependientes, son la mayoría (GiraldoRodríguez et al., 2018).

Además, los cuidadores con sobrecarga tienen menor adherencia a intervenciones de ayuda y le encuentran menos sentido a su actividad de brindar cuidados (Turró Garriga et al., 2017).

Tomando en cuenta este panorama, a continuación se realizará un recuento y una descripción metodológica de diversas estrategias de intervención que han sido propuestas para apoyar a las y los cuidadores informales, con el objeto de conocer sus niveles de eficiencia.

En la revisión sistemática sobre intervenciones de ayuda a cuidadores de personas con demencia realizada por Bustillo et al. (2018), ya referida más arriba, se señala que es a partir de la década de 1970 cuando se desarrollaron diferentes tipos de intervenciones dirigidas a reducir el 
malestar de los cuidadores. El formato más usado ha sido, y continúa siendo desde entonces, el grupal.

El modelo teórico implementado más frecuentemente en estas intervenciones es el de estrés adaptado al cuidado, que implica minimizar las consecuencias negativas a través de estrategias dirigidas a optimizar los recursos personales de los cuidadores. Así lo señala un estudio español que analizó las intervenciones de ayuda para individuos que se dedican a cuidar, y el cual concluye que es importante que la creación de dichas intervenciones retome los avances y los hallazgos propuestos por programas de investigación formales (Losada et al., 2007).

Las intervenciones que se llevan a cabo con mayor frecuencia son: 1) Las intervenciones de respiro, en donde se atiende a la persona dependiente facilitando que el cuidador disponga de algún tiempo libre; 2) Los grupos de autoayuda, en donde un cuidador proporciona consejos y ayuda a otros cuidadores; 3) Los programas psicoeducativos, en donde un profesional entrena a los cuidadores en estrategias y habilidades para afrontar las consecuencias del cuidado; 4) Los programas psicoterapéuticos, en donde se presta especial atención al desarrollo y mantenimiento de una relación terapéutica con los cuidadores (Losada et al., 2007).

No todas las intervenciones presentan el mismo grado de eficacia (o no informan sobre los métodos para medirla), siendo las de tipo psicoeducativo y psicoterapéutico, especialmente las basadas en modelos de estrés y afrontamiento o en modelos cognitivos-conductuales, las que permiten obtener mayores beneficios, pero a condición de que se fomente la participación decididamente activa y comprometida por parte de los cuidadores (Losada et al., 2007; Turró Garriga et al., 2017).

Aún no es posible concluir, puesto que no existen revisiones sistemáticas al respecto, si existe una diferenciación en el nivel de eficacia cuando la intervención involucra a distintos tipos de cuidadores, por ejemplo: a cónyuges en contraste con hijos de personas dependientes. De la misma forma, el sexo del cuidador podría suponer una variable de criba sobre los efectos de una intervención, pero todavía no se comprueban fehacientemente dichos resultados (García et al., 2016).

Quizá cabría pensar que aquellos cuidadores que no mostraran signos de algún malestar emocional (por ejemplo, que no padecieran depresión), obtendrían nulo provecho de intervenciones principalmente diseñadas para reducir patologías y, sin embargo, sí se beneficiarían de intervenciones con un carácter fundamentalmente preventivo, pero tampoco existen suficientes datos para concluir algo terminante sobre el particular (Losada et al., 2007).

Un estudio español sobre las consecuencias del estrés crónico en el funcionamiento cognitivo de las y los cuidadores informales, señala que variables tales como la autoeficacia o los pensamientos disfuncionales son mediadoras relevantes en la reducción de la sintomatología depresiva en las personas que cuidan (Mesa-Gresa et al., 2017).

$\mathrm{Al}$ parecer, los efectos de las intervenciones son mejores cuanto mayor sea el cambio en la percepción de autoeficacia, y cuanto menor resulte 
el número de pensamientos desadaptativos que generan los propios cuidadores (Losada et al., 2007).

Reforzando lo asentado en el párrafo previo, se asegura que las y los cuidadores que tienen mejor salud aplican estrategias de resolución de problemas, de acuerdo con una investigación española que correlacionó índices de bienestar y género (Moya-Albiol \& Moya-Albiol, 2012).

También se ha reportado que la utilización por parte de los cuidadores de estrategias de afrontamiento que tienen que ver con la planificación de actividades, está directamente asociada a su fortaleza psicológica, en tanto que estas estrategias implican la resolución de situaciones prácticas. La correlación anterior puede ocurrir a condición de que exista una red de apoyo social suficiente y significativa, que no deje solos a los y las cuidadoras en sus actividades de asistencia (García et al., 2016).

Seguidamente, se presenta una tabla comparativa de estrategias de atención a personas dependientes:

\section{Tabla 1}

Objetivos, acciones, aspectos positivos y negativos de las estrategias de atención a personas dependientes

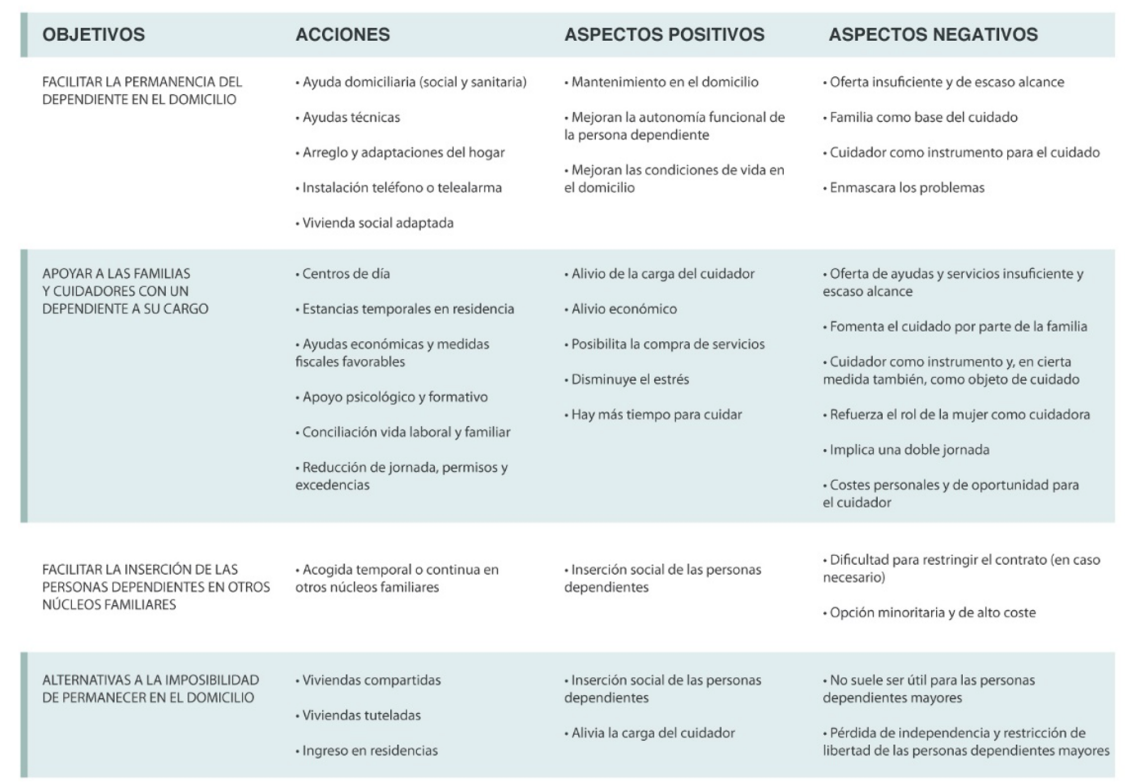

Tabla 1

Escuredo (2007, p. 71).

En la Tabla 1 se esquematizan una serie de estrategias. De entre ellas, las tácticas que apoyan directamente a los cuidadores informales implican -todas- el separar a la persona dependiente del núcleo familiar ya sea temporal o permanentemente. La Tabla 1 se presenta para mostrar que no hay una sola intervención que sea infalible. Sin embargo, los pros y los contras de cada estrategia apuntan a que la mejor metodología de ayuda consiste en (re)conocer las circunstancias y necesidades particulares en cada díada "cuidador informal-persona dependiente". 
Por último, se consigna que existe una amplia variedad de temas y subtemas derivados de las intervenciones de apoyo a cuidadores, que no han sido desarrollados a profundidad, por ejemplo: 1 . Cuidado informal comunitario. 2. Duelo «anticipado» del cuidador por un dependiente. 3. Dinámicas familiares que interfieren con el cuidado. 4. Interacciones psicológicas en el trinomio cuidador-paciente-médico (Losada et al., 2007).

\section{Discusión}

En México, las y los cuidadores informales son un recurso imprescindible para subsanar la demanda de atención a la salud de personas dependientes: todavía no existen aprovisionamientos públicos, privados, o comunitarios capaces de substituir a las personas que cuidan. Pero tampoco parecen ser el objeto de ayuda especializada. Al parecer, ni siquiera despiertan la suficiente curiosidad científica como para ser estudiados seriamente por un programa de investigación longitudinal con revisiones sistemáticas estandarizadas.

En los países que integran la Unión Europea (UE), se está buscando legislar iniciativas de ayudas gubernamentales orientadas a que los empleadores puedan suprimir jornadas partidas a aquellos trabajadores que gestionen cargas familiares de cuidado superiores a las 20 horas semanales. Otras iniciativas de ley proponen otorgar mayor permisividad y flexibilidad horaria, reducir jornadas, o promocionar el teletrabajo en aquellos sectores en los que sea posible hacerlo (Vázquez et al., 2010). Quizá esto pueda implementarse en México a largo plazo.

Mientras llega ese día, hoy por hoy, las y los cuidadores están lidiando con los estragos de la COVID-19. Un muy reciente estudio llevado a cabo en los Estados Unidos de Norteamérica (Czeisler et al., 2020), reseña que un $66 \%$ de cuidadores informales de adultos mayores reportaron tener al menos un síntoma adverso de salud física o mental, durante los 30 días anteriores a la realización de una encuesta telefónica llevada a cabo en junio de 2020, es decir: en plena pandemia.

El mismo estudio contrasta esta cifra con el 6.3\% de personas que en dicha encuesta reportaron tener al menos un síntoma físico o mental adverso, pero que no estaban cuidando a algún individuo en estado de dependencia (Czeisler et al., 2020).

La investigación arriba citada también reporta que $30.7 \%$ de los cuidadores informales norteamericanos encuestados aseguraron haber pensado seriamente en el suicidio durante la pandemia (Czeisler et al., 2020).

Este porcentaje de pensamiento suicida es más alto que el reportado, en el mismo estudio norteamericano, por diferentes grupos de riesgo, tales como: obreros y trabajadores esenciales $=21.7 \%$, jóvenes de 18 a 24 años $=25.5 \%$, hispanos $=18.6 \%$, y personas de raza negra $=15.1 \%$ (Czeisler et al., 2020). 
Acto seguido, y teniendo en la mira la finalización del presente escrito, resulta oportuno exponer un sumario de los principales datos y evidencias encontrados a lo largo de su desarrollo.

En primer término, se manifiesta que el descanso, la economía, el apoyo psicológico, el compatibilizar cuidado y trabajo, la capacitación sobre enfermedades, y la empatía de instituciones y no-cuidadores, son las necesidades más apremiantes de las personas que cuidan (Escuredo, 2007; Montes et al., 2016).

En términos de eficacia se ha comprobado (Bustillo et al., 2018; Losada et al., 2007; Turró Garriga et al., 2017), que las mejores estrategias de intervención para cuidadores se presentan en formatos de modalidad grupal, con dinámicas de participación categóricamente activa, y están basadas en premisas teóricas cognitivo-conductuales.

Sin embargo, sigue siendo una asignatura pendiente la difusión, la disponibilidad, y la facilidad de acceso a estas estrategias de ayuda. Una iniciativa para remediar dicha carencia consiste en la creación de programas de intervención "contextualizados" que se adapten a las necesidades específicas de las y los cuidadores, que alcancen sus objetivos en el menor número de sesiones posible, que se apoyen en metodologías probadas como eficaces, que sean accesibles en términos de tiempo y dinero, y que sean atractivos para clínicos, investigadores y especialistas (Vázquez et al., 2010).

Por tanto, se hace la propuesta de que todas las intervenciones de ayuda a cuidadores informales incluyan en su diseño la evaluación formal de seis áreas, para que sus resultados puedan ser comparados entre sí y evaluados en su eficacia para alcanzar objetivos establecidos de manera previa, con estrategias metodológicas. Estas seis áreas giran alrededor de la persona de las y los cuidadores informales, y son: 1) Salud mental. 2) Salud física. 3) Capacidad de organización. 4) Disposición para buscar ayuda. 5) Capacitación teórico-técnica. 6) Logística económica. Dichas áreas son propuestas por sobre otras, ya que engloban las necesidades más perentorias de los cuidadores de acuerdo con lo reseñado en el presente escrito, y forman un marco que encuadra el contexto particular en el que se desenvuelve cada persona que se dedica a gestionar cuidados a un dependiente.

\section{Conclusión}

Se hace constar que el objetivo de este ensayo consistió en hacer una revisión de las condiciones, circunstancias, necesidades e impactos psicosociales en los cuidadores informales, derivados de su actividad de procurar asistencia a un dependiente. También se revisó la eficacia de algunas estrategias de intervención diseñadas para apoyarles.

La idea básica que sostiene ambas examinaciones se fundamenta en que, al conocer su difícil y vital labor, las personas no-cuidadoras puedan hacer conciencia de los diferentes tipos de ayuda que es posible prestar a quienes están dedicados a atender a otros. Por añadidura, se pretende con estas 
líneas que las y los cuidadores informales sean capaces de afirmarse en su propia identidad, y luego, se procuren mayor y mejor guarda a sí mismos.

Cuidar a otra persona es una habilidad que se aprende a lo largo de nuestra vida. Quizá es la actividad más humana que practicamos. Probablemente es también la actividad que más nos humaniza. Velar por alguien resulta ser una insospechada prueba de fortaleza mental, de equilibrio afectivo, de resistencia física, de flexibilidad familiar, y de resiliencia personal. Cuando amparamos a un individuo en necesidad, también fortalecemos nuestra capacidad de forjar lazos interpersonales afectivos, sanos, fuertes y agradecidos.

En el momento presente, al mirar por alguien más, estamos sentando las bases para ser asistidos en el futuro. Preservar el bienestar de otro ser humano enseña, también, a estimarse uno mismo.

Por todo lo expresado con anterioridad, al término de este ensayo no resulta muy arduo colegir que, por derroteros poco convencionales e inesperados, la actividad de cuidar a otra persona se convierte en un camino idóneo para encontrar nuestro propio bienestar.

\section{Referencias}

Agulló Tomás, M. S., Zorrilla Muñoz, V., \& Gómez García, M. V. P. (2019). Aproximación socio-espacial al envejecimiento y a los programas para cuidadoras/es de mayores. International Journal of Developmental and Educational Psychology, 2(1), 211-231.

Barragán Solis, A. (2007). El género de los cuidadores en la atención del dolor crónico. Estudios de Antropología Básica, 13(2), 1083-1102.

Bustillo, M., Gómez-Gutiérrez, M., \& Guillén, A. I. (2018). Los cuidadores informales de personas mayores dependientes: una revisión de las intervenciones psicológicas de los últimos diez años. Clínica y Salud, 29(2), 89-100. http://dx.doi.org/10.5093/clysa2018a13

Czeisler, M. É., Lane, R. I., Petrosky, E., Wiley, J. F., Christensen, A., Njai, R., \& Rajaratnam, S. M. (2020). Mental health, substance use, and suicidal ideation during the COVID-19 pandemic-United States. Morbidity and Mortality Weekly Report, 69(32), 1049-1057. http://doi.org/10.15585/ mmwr.mm6932al

Díaz, M., Estévez, A., Momeñe, J., \& Ozerinjauregi, N. (2019). Love attitudes and violence: Consequences of burden of care on women. Clinical and Health, 30(3), 123-129. https://doi.org/10.5093/clysa2019a16

Escuredo, B. (2007). Las políticas sociales de dependencia en España. Revista Española de Investigaciones Sociológicas, 119, 65-89.

García, F., Manquián, E., \& Rivas, G. (2016). Bienestar psicológico, estrategias de afrontamiento y apoyo social en cuidadores informales. Psicoperspectivas, 15(3), 87-97. https://doi.org/10.5027/Psicoperspectiva s-Vol15-IssueE3-Fulltext-789

Giraldo-Rodríguez, L., Guevara-Jaramillo, N., Agudelo-Botero, M., Mino-León, D., \& López Ortega, M. (2018). Qualitative exploration of the experiences of informal care-givers for dependent older adults in Mexico City. Ageing \& Society, 39(11), 2377-2396. https://doi.org/10.1017/S0144686X180 00478 
Gutiérrez Cuéllar, P. C. (2019). Percepciones, imágenes y opiniones sobre la vejez desde la mirada de los adultos y jóvenes en México. Espiral (Guadalajara), 26(75), 197-237. https://doi.org/10.32870/eees.v26i75. 7024

INEGI [Instituto Nacional de Estadística Geografía e Informática] (2020). Estadisticas a propósito del Día Internacional de las Personas de Edad. https://inegi.org.mx/contenidos/saladeprensa/aproposito/2019/ edad2019_Nal.pdf

INEGI [Instituto Nacional de Estadística Geografía e Informática] (2009). Encuesta Nacional sobre Uso del Tiempo 2009. http://www.inegi.org.mx/est/contenidos/Proyectos/encuestas/ho gares/especiales/enut/Default.aspex

INGER [Instituto Nacional de Geriatría] (2019).Envejecimiento y Dependencia: Retos y propuestas para una politica pública basada en evidencia. Foro REDAM. https://www.gob.mx/inapam/es/articulos/cuidadores-y-cuida doras-de-persona-mayores?idiom $=\mathrm{es}$

INMUJERES [Instituto Nacional de la Mujeres] (México). (2008). Mujeres y hombres en México. Instituto Nacional de Estadística, Geografía e Informática, México.

López-Ortega, M., \& Aranco, N. (2019). Envejecimiento y atención a la dependencia en México. Banco Interamericano de Desarrollo/InterAmerican Development Bank.

Losada, A., Márquez-González, M., Peñacoba, C., Gallagher-Thompson, D., \& Knight, B. G. (2007). Reflexiones en torno a la atención a los cuidadores informales de personas con demencia y propuesta de una intervención interdisciplinar. Psicología Conductual, 15(1), 57-76.

Mercedes, L., \& Rivera, J. (2018). El cuidador informal de personas mayores institucionalizadas: análisis de la relación y consecuencias del suministro de apoyo. Trabajo Social Hoy, 85, 65-86. https://doi.org/10.12960/TSH .2018 .0016

Mesa-Gresa, P., Ramos-Campos, M., \& Redolat, R. (2017). Cuidado de pacientes oncológicos: una revisión sobre el impacto de la situación de estrés crónico y su relación con la personalidad del cuidador y otras variables moduladoras. Psicooncología, 14(1), 93-106. https://doi.org/10. 5209/PSIC.55814

Montes, A. M. S., Paredes, L. M. M., \& Pérez, J. A. S. (2016). Evaluación e intervención de la sobrecarga del cuidador informal de adultos mayores dependientes: revisión de artículos publicados entre 1997-2014. Archivos de Medicina (Manizales), 16(1), 144-154.

Moya-Albiol, L., \& Moya-Albiol, N. (2012). El cuidado informal: una visión actual. Revista de Motivación y Emoción, 1, 22-30.

Moya-Martínez, P., Escribano-Sotos, F., \& Pardo-García, I. (2012). La participación en el mercado laboral de los cuidadores informales de personas mayores en España. Innovar, 22(43), 55-66.

Muñoz, Á. S. A. (2019). Ansiedad y autoeficacia en cuidadores de pacientes con Alzheimer. Revista INFAD de Psicologia. International Journal of Developmental and Educational Psychology, 2(1), 259-268. https://doi.or g/10.17060/ijodaep.2019.n1.v2.1438

Pabón Poches, D. K., Galvis Aparicio, M. J., \& Cerquera Córdoba, A. M. (2014). Diferencias entre el apoyo social percibido entre cuidadores informales 
y formales de pacientes con demencia tipo Alzheimer. Psicología desde el Caribe, 31(1), 59-78.

Rangel, Y., Mendoza, A., Hernández, L., Cruz, M., Pérez, M., \& Gaytán, D. (2017). Aportes del enfoque de género en la investigación de cuidadores primarios de personas dependientes. Índex de Enfermería, 26(3), Jul-Sep.

Robledo, L. M. F. G., Botero, M. A., Rodríguez, L. G., Campos, R. H. M., González, J. C. M., Pérez, S. L., \& Dávila, H. E. L., (2016). Hechos y desafios para un envejecimiento saludable en México. Instituto Nacional de Geriatría, México.

Saavedra, M. O., Mastrapa, Y. E., \& Aguilar, A. E., (2020). Una concepción de cuidador familiar de anciano. Revista Cubana de Enfermería, 36(1), 3120.

Salazar-Barajas, M. E., Garza-Sarmiento, E. G., García-Rodríguez, S. N., JuárezVázquez, P. Y., Herrera-Herrera, J. L., \& Durán-Badillo, T. (2019). Funcionamiento familiar, sobrecarga y calidad de vida del cuidador del adulto mayor con dependencia funcional. Enfermería Universitaria, 16(4), 362-373. https://doi.org/10.22201/eneo.23958421e.2019.4.615

Tello de la Torre, C., \& Vargas Villamizar, O. H. (2020). Género y trabajo en tiempos del COVID-19: una mirada desde la interseccionalidad. Revista Venezolana de Gerencia (RVG), 25(90), 389-393.

Torres - Castro, S., Szlejf, C., Parra - Rodríguez, L., \& Rosas - Carrasco, O. (2018). Association between frailty and elder abuse in community dwelling older adults in Mexico City. Journal of the American Geriatrics Society, 66(9), 1773-1778. https://doi.org/10.1111/jgs.15489

Trujillo Ornelas, K. M. T., \& Martínez, A. I. M. (2019). Programa para disminuir el índice de sobrecarga en cuidadores informales del adulto mayor. INTEGRA2: Revista Electrónica De Educación Especial Y Familia, $1(10), 135-147$.

Turró Garriga, O., Farrés, S., Pérez, A., \& Batlle, P. (2017). Evaluación del efecto del sentido de coherencia en la carga percibida y en la adherencia a un programa psicoeducativo para cuidadores informales de personas dependientes. Revista Española de Geriatría y Gerontología, 53(4), 196-201. https://doi.org/10.1016/j.regg.2017.12.002

Valdés-Corchado, P. V., Hernández, A. R., Moreno, A. P., \& Carrasco, O. R. (2020). Sociodemographic and clinical characteristics of centenarians in Mexico City. BioMed Research International, 2017, 7195801-7195801. h ttps://doi.org/10.1155/2017/7195801

Vázquez, F. L., Otero, P., López, M., Blanco, V., \& Torres, Á. (2010). Un programa breve basado en la solución de problemas para la prevención de la depresión en cuidadores informales de pacientes con demencia: un estudio piloto. Clínica y Salud, 21(1), 59-76. https://doi.org/10.5093/cl2010v2 $\ln 1 \mathrm{a} 6$ 